\title{
Recurrent Pleomorphic Adenoma: a Case Report
}

\author{
Laliytha Kumar Bijai ${ }^{1, *}$, Philips Mathew ${ }^{2}$, Maria Rajathi ${ }^{2}$, Ravi David Austin ${ }^{2}$ \\ ${ }^{1}$ Department of Dentistry, Sree Balaji Medical College and Hospital, Bharath University, Chennai, India \\ ${ }^{2}$ Department of Oral Medicine and Radiology, Rajah Muthiah Dental College, Annamalai University, Chidambaram, India \\ *Corresponding author: lalithadentist@gmail.com
}

Received July 16, 2014; Revised July 20, 2014; Accepted July 24, 2014

\begin{abstract}
Salivary gland tumors are rare, comprising less than 3\% of all neoplasia of the head and neck region. Pleomorphic adenoma, also known as benign mixed tumor is the most common tumor of the salivary gland accounting for $60-80 \%$ of the benign variety. The tumor is almost always solitary and is usually found as a firm, mobile, painless slow growing mass. Multiple or bilateral pleomorphic adenomas are rare. After surgical resection, up to $43 \%$ of patients are at risk of recurrence, even as late as 45 years after the initial surgery. Here we report a case of recurrent pleomorphic adenoma in a 34-year-old male patient on the right side.
\end{abstract}

Keywords: parotid gland, pleomorphic adenoma, parotidectomy

Cite This Article: Laliytha Kumar Bijai, Philips Mathew, Maria Rajathi, and Ravi David Austin, "Recurrent Pleomorphic Adenoma: a Case Report.” International Journal of Dental Sciences and Research, vol. 2, no. 4 (2014): 92-94. doi: 10.12691/ijdsr-2-4-5.

\section{Introduction}

Pleomorphic adenoma is the most common salivary gland tumor, accounts for about $60 \%$ to $80 \%$ of the benign tumors of the salivary glands and for $60 \%$ to $70 \%$ of all the parotid tumors. This tumor is also known as a benign mixed tumor. The incidence of parotid tumor is about 2.4 in 100000/ year. Right side involvement is more common than left side parotid gland. [1] It is also more common in females than males (2:1). [2] It is found more commonly between fifth and sixth decades of life and its occurrence in children is rare $[3,4]$.

In this paper, we present a case of recurrent pleomorphic adenoma in a 34-year-old male patient on the right side.

\section{Case Report}

A 34-year-old male patient reported to the Department of Oral Medicine and Radiology, Rajah Muthiah Dental College, Annamalai University, Chidambaram, Tamil Nadu with a complaint of swelling on the right side of face since 3 years.

Medical history revealed that the patient was operated for pleomorphic adenoma 8 years back. History of recurrence since 3 years which was initially small in size and later progressed to the present size. No history of associated pain and fever. Patient also gave a history of restoration done 6 months back. Family history was not contributory. His vital signs were within normal limits.

Facial asymmetry was evident due to a diffuse swelling on right lower third of the face in the preauricular region (Figure 1). Skin over the swelling revealed scaring. Secondary changes were not evident. Lobules were palpable, three in number, each of which was approximately $1 \mathrm{X} 2 \mathrm{~cm}$ in size, roughly oval in shape, soft in consistency, neither warm nor tender. Pulsations were not visible or palpable. A single submandibular lymph node was palpable on right side, each of which was approximately $0.5 \mathrm{X} 1 \mathrm{~cm}$ in size, roughly oval in shape, firm, tender and mobile.

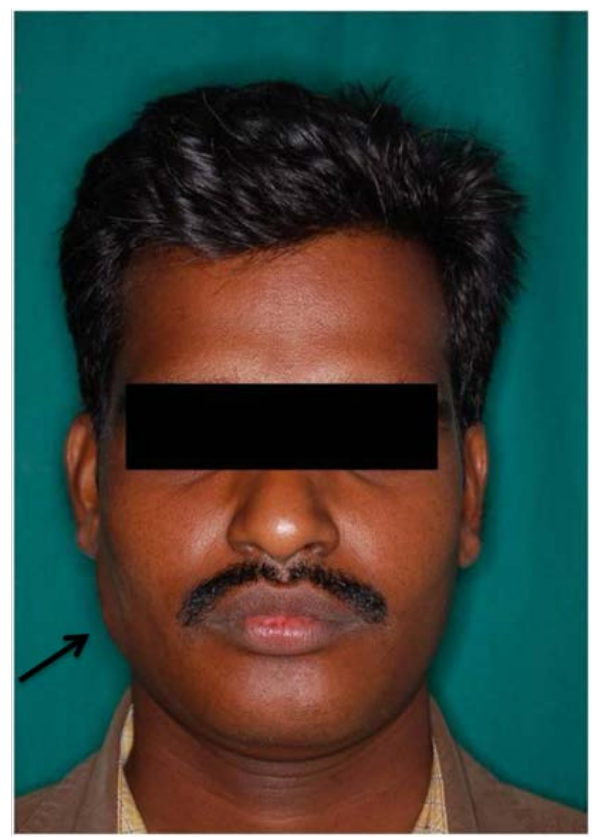

Figure 1. Facial asymmetry due to a diffuse swelling on right lower third of the face

Reports of routine blood investigations revealed raise in polymorphs and erythrocyte sedimentation rate.

Ultrasonogram (USG) scan revealed multiple hypoechoic/ lobulated masses of varying size around 5-10 $\mathrm{mm}$ with internal echoes seen at the level of angle of 
mandible. Vascular uptake was evident only around the periphery (Figure 2).

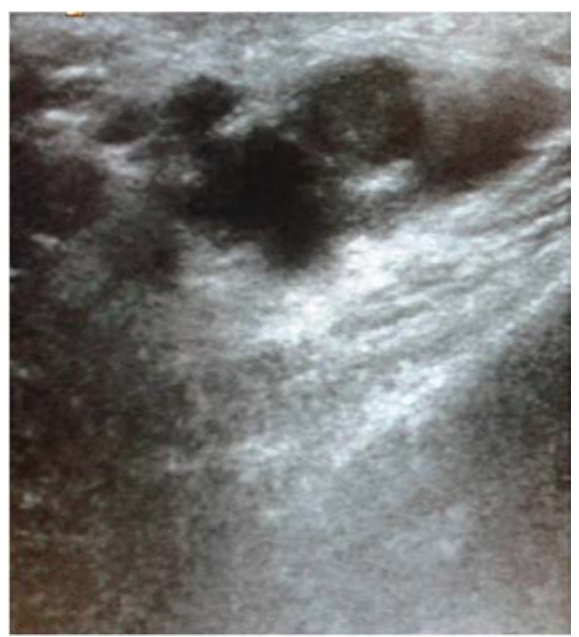

Figure 2. USG scan revealed multiple hypoechoic masses

Based on the history, clinical examination and investigations the case was provisionally diagnosed as recurrent pleomorphic adenoma. The differential diagnosis considered were Warthin's tumor, low grade mucoepidermoid carcinoma, Lymphoma, Keratocystic odontogenic tumor.

An excisional biopsy was performed and specimen was sent for histopathological investigation. The hematoxylineosin stained sections showed islands, nests and sheets of epithelial cells. In some areas there is ductal differentiation with eosinophilic material in the centre. The tumor shows plasmacytoid differentiation in certain areas and majority of the cells are angulated and spindle shaped. In focal areas the cells exhibits polygonal shape and some clear cells are also evident. The stroma is predominantly hyalinized and also shows myxoid and fibrous tissue. Few inflammatory cells are mixed up with tumor cells and the tumor is highly cellular with few blood vessels (Figure 3). These findings were suggestive of pleomorphic adenoma. Superficial parotidectomy was performed and patient is under follow up.

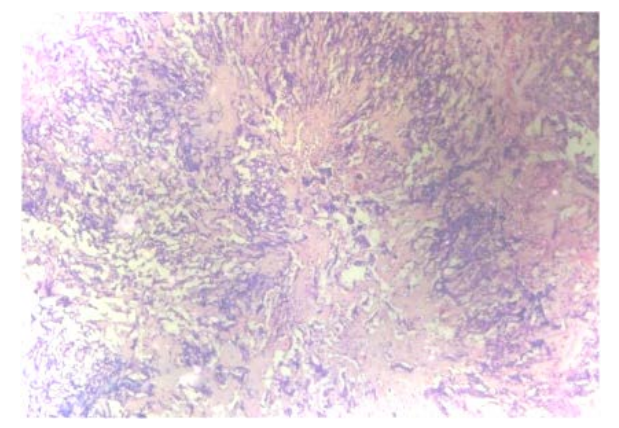

Figure 3. Histopathological section showed islands, nests and sheets of epithelial cells

\section{Discussion}

Pleomorphic adenoma, also known as benign mixed tumor, is the most common tumor of the salivary glands. The typical parotid tumor is found below the lobule of the ear and overlying the angle of the mandible. Usually they are found as a solitary, unilateral, firm, mobile, slow growing asymptomatic mass. On gross specimen, the tumor may be whitish-tan to gray to bluish in colour. It may vary from a few millimetres or centimetres to a giant size. They are irregularly shaped and have a bosselated surface [1].

Signs and symptoms of pleomorphic adenoma depend on the location. When the tumor occurs in the parotid gland, signs of facial nerve weakness are seldom encountered. However, in large neglected tumors, facial nerve weakness is likely to arise as the result of malignant change. The incidence of malignant transformation ranges from 1.9 - 23.3\%. [3] In this case, patient reported with a lobulated swelling on the right parotid region.

The term "Pleomorphic" refers to both histogenesis and histology of the tumor [5].

The tumor has 3 components: an epithelial cell component; a myoepithelial cell component; and a stromal (mesenchymal) component. The identification of these 3 components, which may vary quantitatively from one tumor to another, is essential to the recognition of pleomorphic adenoma. Fine-needle aspiration biopsy can be done for diagnosis of pleomorphic adenoma. ${ }^{[6]}$ Histologically pleomorphic adenoma presents with variable pattern of epithelium in a loosely fibrous stroma, which may be myxoid, chondroid, or mucoid. The epithelium is usually arranged in sheets or strands and ductal structure, often bilayered, are atypical. The myoepithelial cells are often polygonal with a pale eosinophilic cytoplasm.

Several factors have been associated with recurrence. First, a strong relationship exist between the surgical technique and recurrence rate. [7] Enucleation yielded the highest recurrence rates, whereas superficial or total parotidectomy was associated with low recurrence rates. ${ }^{[8]}$ Overall, the recurrence rate was estimated to be between $8 \%$ and $45 \%$ after enucleation and between $2 \%$ and $5 \%$ after superficial parotidectomy, and less than $0.4 \%$ after total parotidectomy. [7] Second, tumor spillage during surgery, due to capsular rupture, was also an important factor for determining recurrence. [9] In this case, patient had a similar swelling 8 years back and patient underwent treatment for the same. Patient has the recurrent swelling since 3 years.

Pleomorphic adenomas do not have true capsules. Rather, they have pseudocapsules with small protrusions, pseudopodia, extending into the surrounding normal parotid gland tissue. [10] In 1998, Henriksson et al. showed an increased incidence of pseudopodia in primary pleomorphic adenomas that subsequently recurred [11].

Presumably, during enucleation, as the main tumor mass is being resected, pseudopodia are left in the gland, giving rise to multiple nodular recurrences. This presumption would explain the increased incidence of recurrence with removal of tumor alone without a "cuff" of normal surrounding gland.

Radiotherapy after surgery has been used for incompletely resected tumors and after surgery complicated with tumor spillage. [12] However, the use of radiotherapy for a benign neoplasm is controversial because of the increased risk for malignant transformation. Moreover, such therapy can result in long-term morbidity, such as hearing loss. Recurrent disease usually occurs late; therefore, some authors claim that follow-ups of less than 10 years are meaningless when reporting a lack of 
recurrence. [9] It is important to control disease at the onset because of the high rate of recurrent tumors and low, but significant, risk of malignant transformation. [13] When evident, recurrent tumors may require repeated surgery, potentially increasing the risks for facial nerve injury and disfiguring outcome. At the time of the second surgery, approximately half the recurrences are multiple (ranging from $46 \%$ to $67 \%$ ).

Therefore, we conclude that when multiple masses are seen in the parotid gland or in the region of the parotid gland, recurrent pleomorphic adenoma is likely. Because the time of recurrence may be as long as 45 years, a history of a parotid gland tumor resection can be blurred. [9] The diagnostician should be aware of this characteristic appearance and raise the possibility of recurrent pleomorphic adenoma even without an adequate history from the patient.

\section{References}

[1] Silva S.J, Costa GT, Filho ACB, Faria PR, Loyola AM Metachronous bilateral pleomorphic adenoma of the parotid gland. Oral surgery, Oral Medicine, Oral Pathology, Oral Radiology and Endodontology. 2006; 101 (3): 333-38.

[2] Shashinder S, Tang IP, Velayutham P, Prepageran N, Gopala KG, Kuljit $S$ et al. A review of parotid tumors and their management: A ten-year-experience. Med J Malaysia 2009; 64 (1): 31-33.
[3] Jr Takahama A, Perez DEC, Magrin J Almeida OP, Kowalski LP. Giant pleomorphic adenoma of the parotid gland. Med Oral Patol Oral Cir Bucal 2008; 13 (1): E 58-60.

[4] Lazard DS, Baujat B, Wagner I, Chabolle F. Surgery in superficial-lobe pleomorphic parotid adenoma. Fr ORL 2005; 87: 59-65.

[5] Kondo T. A case of lipomatous pleomorphic adenoma in the parotid gland: a case report. Diagnostic Pathology 2009; 4: 16-18.

[6] Rodriguez KH, Vargas S, Caroline R, Perez-Atayde A, Shamberger R, McGill TJ, et al. Pleomorphic adenoma of the parotid gland in children. Int J Ped Otorhinolaryngeology 2007; 71 1717-23.

[7] Woods JE, Chong GC, Beahrs OH. Experience with 1,360 primary parotid tumors. Am J Surg 1975; 130: 460-62.

[8] Maynard JD. Management of pleomorphic adenoma of the parotid. Br J Surg 1988; 75: 305-08.

[9] Phillips PP, Olsen KD. Recurrent pleomorphic adenoma of the parotid gland: report of 126 cases and a review of the literature. Ann Otol Rhinol Laryngol 1995; 104: 100-04.

[10] Leverstein H, van der Wal JE, Tiwari RM, van der Waal I, Snow GB. Surgical management of 246 previously untreated pleomorphic adenomas of the parotid gland. Br J Surg 1997; 84: 399-403.

[11] Henriksson G, Westrin KM, Carlsöö B, Silfverswärd C. Recurrent primary pleomorphic adenomas of salivary gland origin. Cancer 1998; 82: 617-20.

[12] Gleave EN, Whittaker JS, Nicholson A. Salivary tumors: experience over thirty years. Clin Otolaryngol 1979; 4: 247-57.

[13] Som PM, Shugar JMA, Sacher M, Stollman AL, Biller HF. Benign and malignant parotid pleomorphic adenomas: CT and MR studies. J Comput Assist Tomogr 1988; 12: 65-9. 